\title{
Stellar population gradients in GASS
}

\author{
Jonas Johansson $^{1}$, Guinevere Kauffmann ${ }^{1}$ and Sean Moran ${ }^{2}$ \\ ${ }^{1}$ Max-Planck Institut für Astrophysik, Karl-Schwarzschild-Str. 1, \\ D-85741 Garching, Germany \\ ${ }^{2}$ Department of Physics and Astronomy, The Johns Hopkins University, \\ 3400 N. Charles Street, Baltimore, MD 21218, USA
}

\begin{abstract}
We study relationships between the stellar populations and interstellar medium in massive galaxies using the Galex Arecibo SDSS Survey (GASS). The sample consists of HIobservations ( $\sim 1000$ galaxies) and complementary $\mathrm{H}_{2}$-observations (330 galaxies) and long-slit spectroscopy (230 galaxies). Luminosity-weighted stellar population ages, metallicitites and element abundance ratios, are derived by fitting stellar population models of absorption line indices. We find that the ages correlate more strongly with molecular gas fraction $\left(\mathrm{M}_{H_{2}} / \mathrm{M}_{*}\right)$ than with neutral Hydrogen fraction $\left(\mathrm{M}_{H I} / \mathrm{M}_{*}\right)$. This result strengthens the theory that $\mathrm{H}_{2}$ is a better tracer of star-formation than HI. The sample is dominated by negative metallicity-gradients and flat $\mathrm{Mg} / \mathrm{Fe}$-gradients. Galaxies with high $\mathrm{M}_{H_{2}} / \mathrm{M}_{*}$-ratios show in general flat or weakly negative age-gradients. For low $\mathrm{M}_{H_{2}} / \mathrm{M}_{*}$-ratios the age-gradients are overall negative. These results are in agreement with the inside-out galaxy formation scenario. For galaxies with high $r_{90} / r_{50}$ ratios, a sub-population show positive age-gradients indicating additional formation channels. Furthermore, for galaxies with high $\mathrm{M}_{H_{2}} / \mathrm{M}_{*}$-ratios more massive systems have older stellar populations in their centers, suggesting downsizing within the inside-out formation scenario.
\end{abstract}

Keywords. galaxies: evolution - galaxies: elliptical and lenticular, $\mathrm{cD}$ - galaxies: spiral - galaxies: stellar content

\section{Introduction}

The sizes of galaxies, i.e., the effective radii, have been found to increase with decreasing redshift, suggesting an inside-out formation scenario (van Dokkum et al. 2010). Trujillo et al. (2007) show that this occurs for both disc galaxies as well as spheroids for redshifts up to $z \sim 2$. A possible mechanism for size growth is minor merging. Szomoru et al. (2012) found that this mechanism is responsible for size evolution of elliptical galaxies since $z \sim 2$ through the accretion of satellite galaxies, adding stellar content to the outskirts of the galaxies. Marmol-Queralto et al. (2013) find that satellites typically have younger stellar ages than the central galaxies. Hence, the accretion of such systems would produce negative stellar age-gradients. On the other hand, several authors have found flat or positive age-gradients in elliptical galaxies (e.g. La Barbera et al. 2012).

Cold gas accretion is an additional way of driving galaxy growth, e.g. Dekel \& Birnboim (2006) predict disc growth through cold streams. Kauffmann et al. (2006) found starformation in regions of high stellar density, fed by cold gas accretion. Wuyts et al. (2012) also found enhanced star-formation in regions of high stellar density, located at off center radii and consequently older stellar ages in the centers of star-forming galaxies. These results indicate negative age-gradients in the cold gas accretion scenario.

In this work we derive stellar population gradients, including age, metallicity and element abundance ratios, for a sample of 182 galaxies drawn from the GALEX Arecibo SDSS Survey (GASS, Catinella et al. 2010). This allows us to study the stellar population gradients in relation to neutral and molecular Hydrogen mass fractions for an unbiased sample of massive galaxies. 


\section{Data}

\subsection{GASS, COLD GASS and long-slit spectroscopy}

GASS comprises observations of the $21 \mathrm{~cm}$ line of neutral Hydrogen, obtained with the Arecibo radio telescope. In the final stage, observations for an unbiased sample of $\sim 1000$ galaxies, in the redshift range $0.025<z<0.05$ and with stellar masses greater than $10^{10} \mathrm{M}_{\odot}$, will be gathered. The sample was selected using the Sloan Digital Sky Survey (SDSS) spectroscopic and Galaxy Evolution Explorer (GALEX) imaging surveys.

In addition to GASS, COLD GASS (Saintonge et al. 2011) constitutes molecular gas masses determined through observations of the CO $(\mathrm{J}=1-0)$ line using the IRAM $30 \mathrm{~m}$ telescope. COLD GASS is a sub-sample of GASS and consists of 330 galaxies. Furthermore, Moran et al. (2012) obtained long-slit spectroscopy for a sub-sample of 182 galaxies with the Blue Channel Spectrograph on the $6.5 \mathrm{~m}$ MMT telescope on Mt. Hopkins, AZ, and 51 galaxies at the Apache Point Observatory (APO). In this work we focus on the former observations that were obtained through a 1.25 " wide slit in the wavelength range 3900-7000 $\AA$ and at a spectral resolution of $\sim 4 \AA$ FWHM. Each two-dimensional, rotation-curve corrected spectrum has been binned adaptively in the spatial direction until a signal-to-noise $(\mathrm{S} / \mathrm{N})$ of 15 is typically reached for a maximum bin size of 3 ". These limits were set to change with radius to ensure a balance between $\mathrm{S} / \mathrm{N}$ and bin size for each co-added spectrum.

\subsection{Stellar population parameters}

We utilise the SED-fitting code GANDALF (Sarzi et al. 2006), which is based on the penalised pixel-fitting (PPXF) method of Cappellari \& Emsellem (2004), to obtain clean absorption spectra. These are used for deriving the Lick absorption line indices (Worthey \& Ottaviani 1997; Trager et al. 1998), defined for 25 prominent absorption features in the optical. The resolution of the spectra are downgraded to the Lick/IDS resolution $(\sim 8-10$ $\AA$, Worthey \& Ottaviani 1997) prior to measuring the indices. The index measurements are corrected for velocity dispersion broadening.

From the derived Lick indices we determine the stellar population parameters age and total metallicity and the element abundance ratios accessible through integrated light spectroscopy of galaxies $(\mathrm{O} / \mathrm{Fe}, \mathrm{Mg} / \mathrm{Fe}, \mathrm{C} / \mathrm{Fe}, \mathrm{N} / \mathrm{Fe}, \mathrm{Ca} / \mathrm{Fe}$ and $\mathrm{Ti} / \mathrm{Fe})$. Using the method described in detail in Johansson et al. (2012), we fit the stellar population models of absorption line indices from Thomas et al. (2011). These are single stellar population models with variable element abundance ratios for the 25 Lick indices.
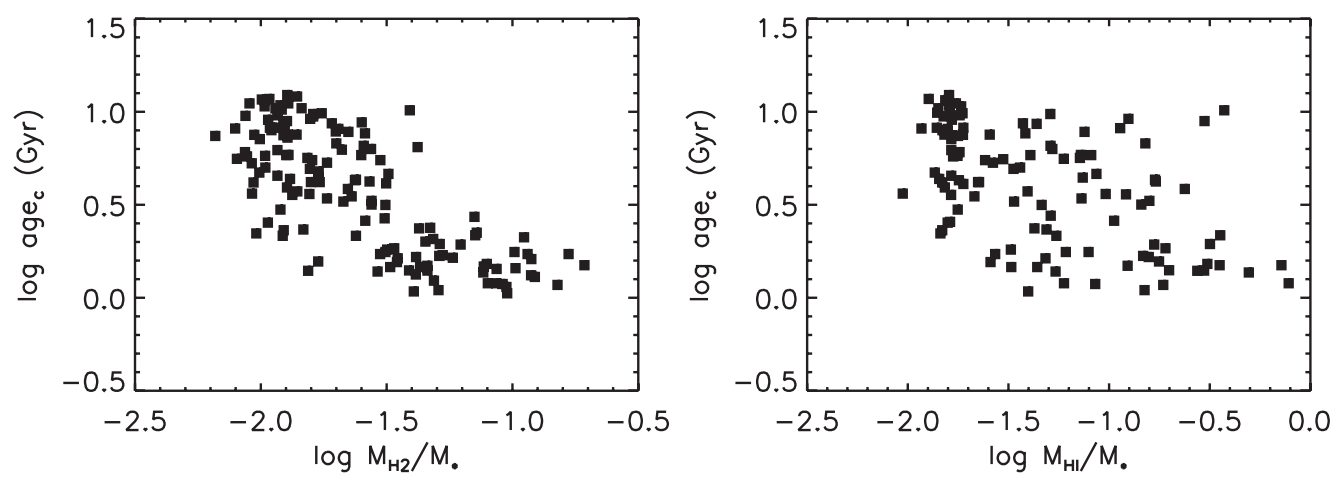

Figure 1. Stellar age within $r<0.4 R_{50}$ versus $M_{H_{2}} / M_{*}$ (left panel) and $M_{H I} / M_{*}$ (right panel). 


\section{Results and Discussion}

We calibrate the Lick indices derived from the long-slit spectra to those derived from the corresponding SDSS spectra and find in general a very good agreement. Derived central stellar population ages from stacked spectra within $r<0.4 r_{50}$ for each galaxy $\left(\operatorname{age}_{c}\right)$ are compared with molecular gas fraction (left panel) and neutral Hydrogen fraction (right panel) in Fig. 1. It is clear that the ages correlate stronger with molecular gas fraction than with neutral Hydrogen fraction. This result strengthens the theory that molecular gas fraction is a better tracer of star formation than neutral hydrogen.

We compute the residuals between the stellar population parameters derived from central stacks $\left(r<0.4 r_{50}\right)$ and those derived from stacked spectra of outer radial bins $\left(r>0.4 r_{50}\right)$. The residuals are compared to the molecular gas fractions and we find in general higher metallicities in the central stacks, suggesting negative metallicity gradients. The $[\mathrm{Mg} / \mathrm{Fe}]$ residuals instead suggest overall flat gradients. Fig. 2 shows the residuals in stellar population age as a function of molecular gas fraction. We find that galaxies with high molecular gas fractions show slightly older stellar ages in the central parts. As we go towards lower gas fractions the spread becomes larger, but the majority of the galaxies show residuals suggesting negative age-gradients. Galaxies with $r_{90} / r_{50}>3$ (filled squares in Fig. 2), i.e., the most bulge-dominated objects, have in general low molecular gas fractions, as expected, and show the largest spread in the age-residuals. This result indicates a wide range of age-gradients and possibly different formation histories.

We further stack the galaxies in three bins of molecular gas fraction together with six radial bins $\left(r / r_{50}\right)$. The derived stellar population parameters for these stacks are shown in Fig. 3. It is clear that the overall stellar ages, metallicities and $\mathrm{Mg} / \mathrm{Fe}$ ratios increase with decreasing molecular gas fraction. It should be noted that the average stellar mass also increase slightly with decreasing molecular gas fraction.

Fig. 3 shows negative luminosity-weighted age-gradients that increase in strength for lower molecular gas fractions. This result is consistent with the inside-out galaxy formation scenario (van Dokkum et al. 2010). We can further see that all bins of molecular gas fraction show steep metallicity gradients, but flat $\mathrm{Mg} / \mathrm{Fe}$-gradients. In the inside-out formation scenario we can understand this if stars keep forming from recycled, in-falling gas with high $\mathrm{Mg} / \mathrm{Fe}$ ratios and lower total metallicities and/or accretion of stellar systems with these characteristics. The bottom right panel of Fig. 3 shows the results from stacking objects in two bins of molecular gas fraction together with two mass-bins. These results indicate that more massive, high molecular gas fraction galaxies have ceased

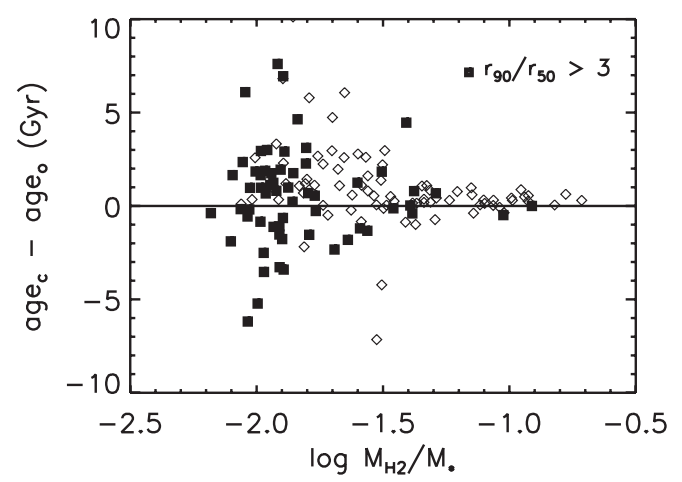

Figure 2. Central stellar age $\left(\right.$ age $\left._{c}, r<0.4 r_{50}\right)$ minus outer stellar age $\left(\right.$ age $\left._{o}, r>0.4 r_{50}\right)$ as a function of $\mathrm{M}_{H_{2}} / \mathrm{M}_{*}$. Solid square are galaxies with $r_{90} / r_{50}>2.5$. 

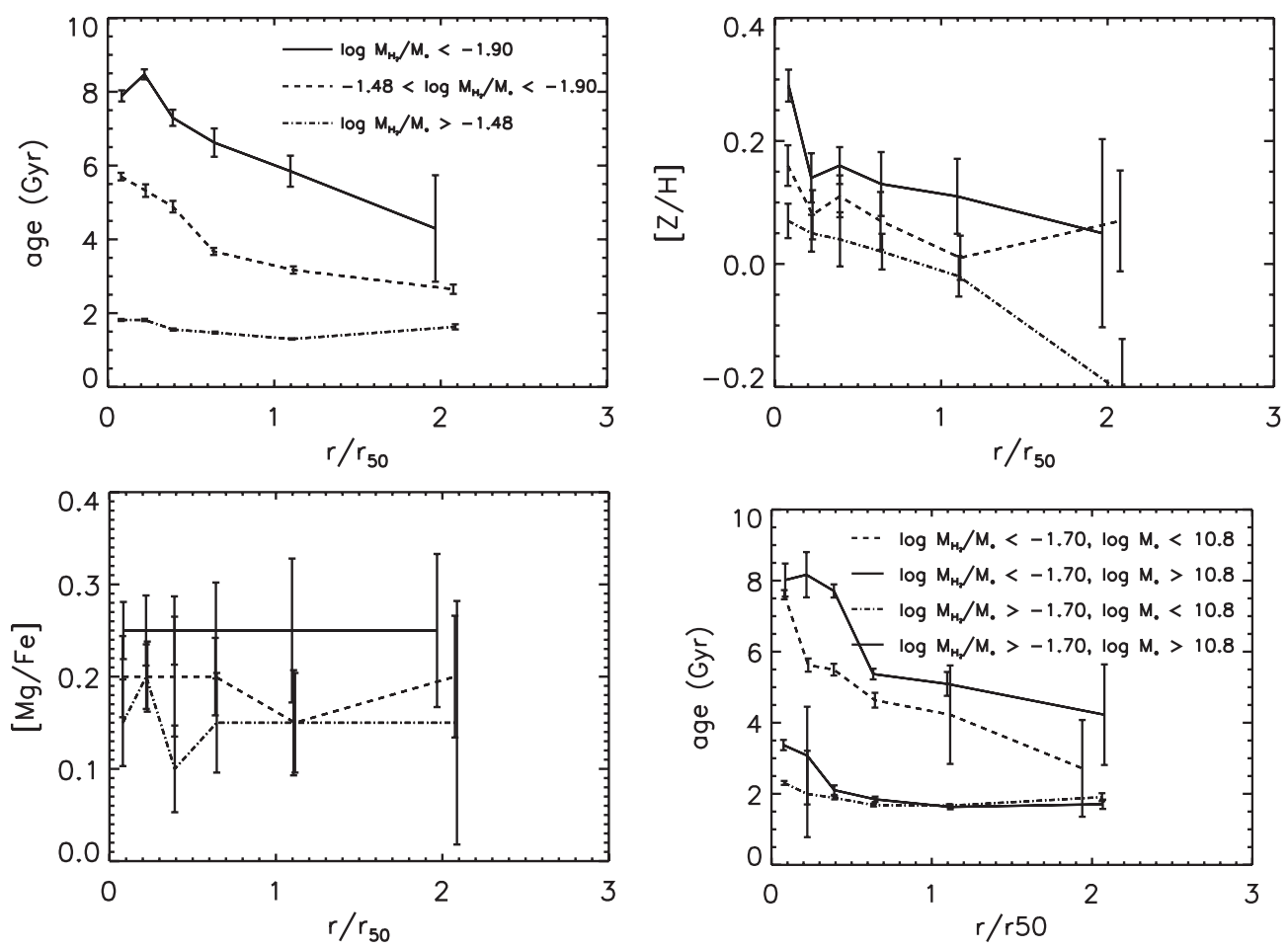

Figure 3. Age- (top left), Z/H- (top right) and Mg/Fe-gradients (bottom left) of stacked galaxies in three bins of $\mathrm{M}_{H_{2}} / \mathrm{M}_{*}$ given by the labels in the top left panel. Bottom right: age-gradients in bins of $\mathrm{M}_{H_{2}} / \mathrm{M}_{*}$ and stellar mass as given by the labels.

forming stars in the central parts, suggesting downsizing within the inside-out formation scenario.

\section{References}

Cappellari, M. \& Emsellem, E., 2004, PASP, 116, 138

Dekel, A. \& Birnboim, Y., 2006, MNRAS, 368, 2

Johansson, J., Thomas, D., \& Maraston, C., 2012, MNRAS, 421, 1908

Kauffmann, G., et al., 2006, MNRAS, 367, 1394

La Barbera, et al., 2012, MNRAS, 426, 2300

Marmol-Queralto, E., Trujillo, I., Villar, V., Barro, G., \& Perez-Gonzalez, P., 2013, MNRAS, 429, 792

Moran, S., et al., 2012, ApJ, 745, 66

Catinella, B., 2010, MNRAS, 403, 683

Saintonge, A., Kauffmann, G., Wang, J., et al. 2011, MNRAS, 415, 61

Sarzi, M., et al., 2006, MNRAS, 366, 1151

Szomoru, D., Franx, M., \& van Dokkum, P., 2012, ApJ, 749, 121

Thomas, D., Maraston, C., \& Johansson, J., 2011, MNRAS, 412, 2183

Trager, S. C., Worthey, G., Faber, S. M., Burstein, D., \& Gonzalez, J. J., 1998, ApJS, 116, 1

Trujillo, I., et al., 2007, MNRAS, 382, 109

van Dokkum, P. G., et al., 2010, ApJ, 709, 1018

Worthey, G. \& Ottaviani, D. L., 1997, ApJS, 111, 377

Wuyts, S., 2012, ApJ, 753, 114 